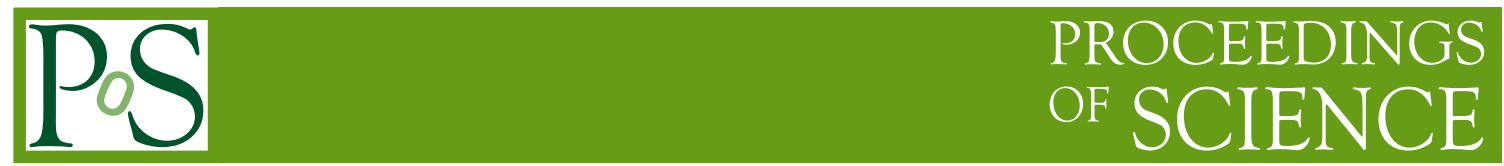

\title{
Long-baseline accelerator neutrino experiments
}

\author{
Atsuko K. Ichikawa* Kyoto University \\ E-mail: ichikawa@scphys.kyoto-u.ac.jp
}

Whole structure of the flavor-mass mixing in the lepton sector is now known through the measurements of neutrino oscillation. Remaining is the CP phase, which is yet totally unknown. One of the mixing angles, $\theta_{23}$, is close to maximal mixing and its precise measurement is important to understand the origin of the flavor-mass mixing. The recent observation of $v_{e}$ appearance by the accelerator-based long-baseline experiment, T2K, opened a way to access the CP phase and $\mathrm{CP}$ violation in the lepton sector. Precise measurement of $\theta_{23}$ and determination of mass ordering are also expected with on-going and future accelerator-based experiment. Current status and prospects of accelerator-based long-baseline neutrino oscillation experiments are reviewed.

Flavor Physics \& CP Violation 2015

May 25-29, 2015

Nagoya, Japan

${ }^{*}$ Speaker. 


\section{Introduction}

Quantum interference has been playing a unique role to examine the properties of quarks and neutrinos. However it appears in quite different scales for these two species. The time evolution of a mass eigenstate $m_{i}$ is given as $e^{-i(E t-p x)}$. Quarks can be treated approximately in a rest frame, hence $e^{-i(E t-p x)} \approx e^{-i m_{i} t}$. This implies that the modulation frequency is determined by mass difference $\Delta m$ and oscillation happens in nano to pico seconds. Neutrinos are ultra-relativistic particle due to its extremely light mass. So $e^{-i(E t-p x)} \approx e^{-i \frac{m_{i}^{2}}{2 E} L}$ and modulation frequency is governed by squared mass difference $\Delta m^{2}$ and the oscillation is observed in milli-second time scale or $100 \mathrm{~km}$ space scale for $\sim 1 \mathrm{GeV}$ neutrinos, which is a typical energy of accelerator-produced neutrinos. The interaction length of a $1 \mathrm{GeV}$ neutrino in a $1 \mathrm{~g} / \mathrm{cm}^{3}$ matter is $10^{8} \mathrm{~km}$, roughly corresponding to the distance between the Earth and the Sun. Therefore, it penetrates the Earth without being lost and reaches $>100 \mathrm{~km}$ away detectors.

Like the case of the quark sector, we now know that the weak flavor eigenstates, $v_{e}, v_{\mu}$, and $v_{\tau}$ are related to the mass eigenstates, $v_{1}, v_{2}$ and $v_{3}$, by the unitary mixing matrix $U$ :

$$
\left(\begin{array}{c}
v_{e} \\
v_{\mu} \\
v_{\tau}
\end{array}\right)=\left(\begin{array}{ccc}
U_{e 1} & U_{e 2} & U_{e 3} \\
U_{\mu 1} & U_{\mu 2} & U_{\mu 3} \\
U_{\tau 1} & U_{\tau 2} & U_{\tau 3}
\end{array}\right)\left(\begin{array}{c}
v_{1} \\
v_{2} \\
v_{3}
\end{array}\right)
$$

It is called the Pontecorvo-Maki-Nakagawa-Sakata (PMNS) matrix, and is commonly parameterized as

$$
U_{P M N S}=\left(\begin{array}{ccc}
1 & 0 & 0 \\
0 & C_{23} & S_{23} \\
0 & -S_{23} & C_{23}
\end{array}\right)\left(\begin{array}{ccc}
C_{13} & 0 & S_{13} e^{-i \delta_{\mathrm{CP}}} \\
0 & 1 & 0 \\
-S_{13} e^{+i \delta_{\mathrm{CP}}} & 0 & C_{13}
\end{array}\right)\left(\begin{array}{ccc}
C_{12} & S_{12} & 0 \\
-S_{12} & C_{12} & 0 \\
0 & 0 & 1
\end{array}\right)
$$

with $C_{i j}\left(S_{i j}\right)$ representing $\cos \theta_{i j}\left(\sin \theta_{i j}\right)$, where $\theta_{i j}$ is the mixing angle between the generations $i$ and $j$. There is one irreducible phase, $\delta_{\mathrm{CP}}$, allowed in a unitary $3 \times 3$ mixing matrix. Then, one type of a flavor-eigenstate neutrino, $v_{\alpha}$, changes, after traveling a distance $L$, its flavor to $\beta$ with an amplitude:

$$
<v_{\beta} \mid v_{\alpha}(L)>=U_{\beta i}^{*} U_{\alpha i} e^{-i \frac{m_{i}^{2}}{2 E} L}
$$

From measurements of neutrinos from the Sun, reactors, atmosphere and accelerators, we know[1]:

$$
\begin{array}{r}
\theta_{12}=33^{\circ} \pm 1^{\circ}, \theta_{23}=46^{\circ} \pm 3^{\circ}, \theta_{13}=8.9^{\circ} \pm 0.4^{\circ} \\
\Delta m_{21}^{2}=(7.5 \pm 0.2) \times 10^{-5} \mathrm{eV}^{2},\left|\Delta m_{32}^{2}\right|=(2.44 \pm 0.06) \times 10^{-3} \mathrm{eV}^{2} .
\end{array}
$$

The $\theta_{23}$ is now most unknown. It is consistent with $45^{\circ}$, i.e., maximal mixing, within the uncertainty. We also don't know the order of mass, i.e., $m_{1}<m_{2}<<m_{3}$ or $m_{3}<<m_{1}<m_{2}$. The order would have a big impact on the neutrinoless double beta decay search, hence on the confirmation/refutation of the Majorana neutrino hypothesis. Most important unknown parameter is the $\mathrm{CP}$ phase $\delta_{\mathrm{CP}}$. It would cause $\mathrm{CP}$ violation in the lepton sector and can be a key to understand the origin of the matter-dominant universe. The value is totally unknown yet. Current and future 
Table 1: Long-baseline experiments in the world

\begin{tabular}{lccc}
\hline & proton beam energy & proton beam power (planned) & detector mass \\
\hline K2K & $12 \mathrm{GeV}$ & $7 \mathrm{~kW}$ & $50 \mathrm{kt}$ \\
MINOS & $120 \mathrm{GeV}$ & $400 \mathrm{~kW}$ & $5.4 \mathrm{kt}$ \\
OPERA/ICARUS & $400 \mathrm{GeV} / \mathrm{c}$ & $500 \mathrm{~kW}$ & $1.2 \mathrm{kt} / 0.6 \mathrm{kt}$ \\
T2K & $30 \mathrm{GeV}$ & $350 \mathrm{~kW}(750 \mathrm{~kW})$ & $50 \mathrm{kt}$ \\
NOvA & $120 \mathrm{GeV}$ & $420 \mathrm{~kW}(700 \mathrm{~kW})$ & $14 \mathrm{kt}$ \\
\hline
\end{tabular}

accelerator-based long baseline neutrino experiments are aiming to do a precise measurement of $\theta_{23}$, to determine the mass ordering and to explore $\mathrm{CP}$ violation in the lepton sector.

The experiment is conducted by producing a muon neutrino beam and measuring it a $O(100) \mathrm{km}$ away detector. In more detail, a proton beam from accelerators is injected to a target and pions are produced. Those pions decay in-flight and produce muon neutrinos. Electromagnetic horns are used to focus the pions in parallel by toroidal magnetic field and enhance neutrinos in the forward direction. By changing the polarity of the horns, either muon neutrino beam or muon antineutrino beam is produced. In case of the T2K experiment, which is introduced later, $\sim 1 \mathrm{v} / \mathrm{cm}^{2} / \mathrm{s}$ penetrate the $295 \mathrm{~km}$ away far detector when the accelerator is operated at a $750 \mathrm{~kW}$ beam power.

The produced muon neutrinos then would change the flavor due to neutrino oscillation with the probability given as:

$$
1-P\left(v_{\mu} \rightarrow v_{\mu}\right)=\sin ^{2} 2 \theta_{23} \sin ^{2}\left(\Delta m^{2} \frac{L}{4 E}\right),
$$

where $\Delta m^{2} \approx m_{3}^{2}-m_{2}^{2} \approx m_{3}^{2}-m_{1}^{2}, L$ is the distance between the neutrino production point and the far detector and $E$ is the energy of the neutrino. Since $\sin ^{2} 2 \theta_{23}$ is close to one, most of muon neutrinos 'disappears'. Among those, some are expected to appear as electron neutrinos with the probability:

$$
P\left(v_{\mu} \rightarrow v_{e}\right)=\sin ^{2} \theta_{23} \sin ^{2} 2 \theta_{13} \sin ^{2}\left(\Delta m^{2} \frac{L}{4 E}\right) .
$$

Note that these probabilities are leading term and sub-leading terms follow. Recent reactor measurements give $\sin ^{2} 2 \theta_{13}=0.093$ [1], so only about $5 \%$ appears as electron neutrinos and dominant fraction changes into tau neutrinos and appear if energy is above the $\tau$ production threshold $(\sim 3.5 \mathrm{GeV})$.

\section{World Long baseline neutrino oscillation experiments}

The first long baseline neutrino oscillation experiment, K2K, started in 1999 in Japan with a $250 \mathrm{~km}$ baseline. Since then, number of experiments were or are being conducted. Table 1 summarizes those experiments. Most of these experiments intended or are intending to do a precise measurement of $v_{\mu}$ disappearance and to search for or measure $v_{e}$ appearance. 
Only the OPERA and ICARUS experiments have a neutrino beam whose energy is high enough to produce a tau. OPERA used nuclear emulsion as the far detector to tag the tau decay. ICARUS is a first experiment which realized large-scale $(300$ ton $\times 2)$ liquid Ar TPC's.

$\mathrm{T} 2 \mathrm{~K}$ and NOvA are running experiments.

$\mathrm{T} 2 \mathrm{~K}$, in Japan, uses a neutrino beam produced with the J-PARC accelerator at Tokai and detects it with the Super-Kamiokande (SK) detector at Kamioka. The baseline length is $295 \mathrm{~km}$ and the peak energy of the beam is $0.6 \mathrm{GeV}$ where the oscillation probability becomes maximum. The accumulated number of protons on target (POT), which is generally used as the statistics to represent the amount of data taken, is $7.0 \times 10^{20}$ for the neutrino beam and $3.1 \times 10^{20}$ for the antineutrino beam as of June 2015. The experiment is aiming to collect $78 \times 10^{20}$ POT at the end. The Super-Kamiokande is a gigantic water Cerenkov detector. Events are classified with the number of observed Cerenkov rings and types of rings, i.e., a diffuse electron-like ring and a sharp muon-like ring. Single $\mu$-like ring events are signal for $v_{\mu}$ disappearance and single e-like ring events are signal for $v_{e}$ appearance. The separation of $\mu$-like and e-like events is very good: mis-identification probability is about $1 \%$.

NOvA uses a beam from the Main Injector at FNAL. The baseline length is $810 \mathrm{~km}$ and the beam peak energy is $2 \mathrm{GeV}$, which is slightly higher than the oscillation maximum $(\sim 1.6 \mathrm{GeV})$. NOvA's far detector is made of liquid scintillator. Extruded PVC tubes inserted with wavelength shifting fibers made up 344,000 cells and enable both tracking and calorimeter. The $v_{e}$ events or $v_{\mu}$ events are selected with a showering or straight track, respectively.

\section{Muon neutrino disappearance - Is $\theta_{23} 45^{\circ}$ ?-}

The C.L. regions for $\sin ^{2} \theta_{23}$ and $\Delta m_{32}^{2}$ obtained primary from muon neutrino disappearance measurements are shown in Fig. 1[2]. The current most precise result is by T2K and obtained $\theta_{23}$ is $46^{\circ} \pm 3^{\circ}$.

\section{Tau neutrino appearance}

Disappearance of muon neutrinos from atmosphere or accelerators has been considered mainly due to $v_{\mu}$ to $v_{\tau}$ oscillation because only small portion appears as $v_{e}$. This should be confirmed experimentally. The Super-Kamiokande collaboration reported the $v_{\tau}$ appearance signal in the atmospheric neutrino samples with $3.8 \sigma$ significance[3]. This is based on the excess in the enhanced $\tau$-like events over background. The OPERA experiment is aiming to identify the $\tau$ production in the event-by-event basis. So far they observed four $v_{\tau}$ candidate events and $v_{\mu}$ to $v_{\tau}$ oscillation is confirmed with a $4.2 \sigma$ significance[4].

\section{Electron neutrino appearance - Golden mode for CP phase and mass ordering -}

As can be seen in Eq.1.1, the imaginary part vanishes in the probability of disappearance $(\beta=\alpha)$. Hence measurements of appearance of particular type of neutrino are necessary to see $\mathrm{CP}$ violation in neutrino oscillation. Accumulation of tau neutrino appearance events is very difficult. An electron neutrino beam having energy above muon production threshold is not realized so far. 

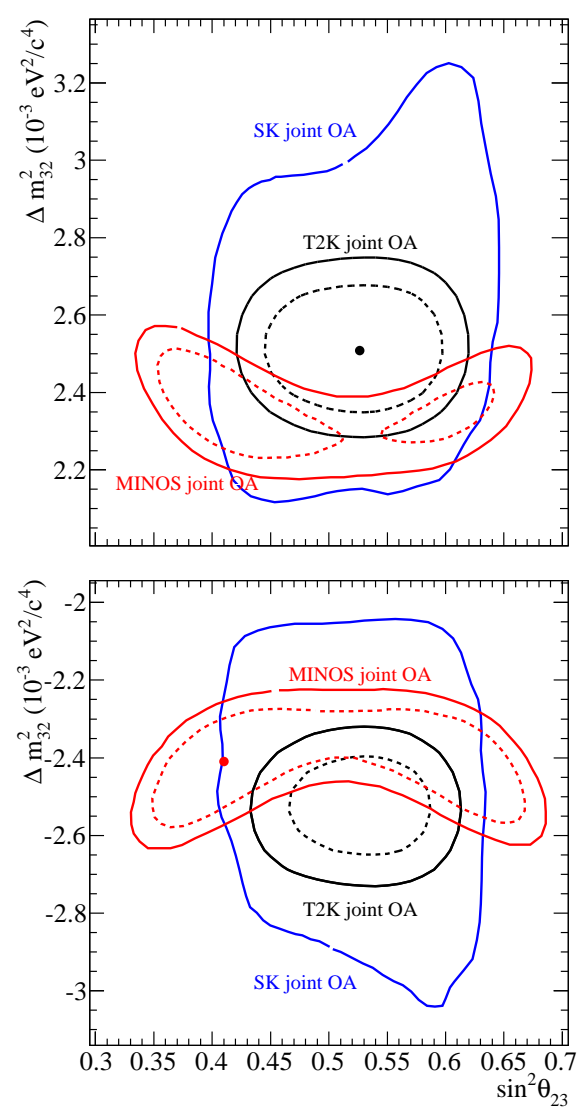

Figure 1: The 68\% (dashed) and 90\% (solid) C.L. regions for normal(top) and inverted(bottom) mass ordering[2]. Those shown are by T2K, MINOS accelerator experiments and by the Super-Kamiokande(SK) atmospheric neutrino measurement.

Therefore, appearance of electron neutrino from muon neutrino is the golden mode or practically a unique way to see $\mathrm{CP}$ violation in the lepton sector. Equation 1.2 shows the leading term only. A more exact $v_{e}$ appearance formula is given as

$$
\begin{aligned}
P\left(v_{\mu} \rightarrow v_{e}\right)= & 4 C_{13}^{2} S_{13}^{2} S_{23}^{2} \sin ^{2} \Phi_{31}\left(1+\frac{2 a}{\Delta m_{31}^{2}}\left(1-2 S_{13}^{2}\right)\right) \\
& +8 C_{13}^{2} S_{12} S_{13} S_{23}\left(C_{12} C_{23} \cos \delta_{\mathrm{CP}}-S_{12} S_{13} S_{23}\right) \cos \Phi_{32} \sin \Phi_{31} \sin \Phi_{21} \\
& -8 C_{13}^{2} C_{12} C_{23} S_{12} S_{13} S_{23} \sin \delta_{\mathrm{CP}} \sin \Phi_{32} \sin \Phi_{31} \sin \Phi_{21} \\
& +4 S_{12}^{2} C_{13}^{2}\left(C_{12}^{2} C_{23}^{2}+S_{12}^{2} S_{23}^{2} S_{13}^{2}-2 C_{12} C_{23} S_{12} S_{23} S_{13} \cos \delta_{\mathrm{CP}}\right) \sin ^{2} \Phi_{21} \\
& -8 C_{13}^{2} S_{13}^{2} S_{23}^{2}\left(1-2 S_{13}^{2}\right) \frac{a L}{4 E_{v}} \cos \Phi_{32} \sin \Phi_{31},
\end{aligned}
$$

where $C_{i j}=\cos \theta_{i j}, S_{i j}=\sin \theta_{i j}, \Phi_{i j}=\Delta m_{i j}^{2} \frac{L}{4 E}$ and $a \equiv 2 \sqrt{2} G_{F} n_{e} E=7.56 \times 10^{5} \mathrm{eV}^{2} \frac{\rho}{g \mathrm{~cm}^{-3}} \frac{E}{\mathrm{GeV}}$ and $\rho$ is the density of matter in which neutrinos propagate. The terms having $a$ originate from the interaction between neutrino and matter and are called the 'matter effect'. The equation includes only the first order for the matter effect. The probability for antineutrino is obtained by flipping the sign of $\delta_{\mathrm{CP}}$ and $a$ so the term with $\sin \delta_{\mathrm{CP}}$ causes difference of the probability and hence $\mathrm{CP}$ 
violation. At around the energy and baseline length where the probability becomes maximum and for $\sin ^{2} \theta_{23}=0.5$, that CP-violating term causes at most $\pm 27 \%$ difference in the oscillation probability. The matter-effect term contributes in the opposite way depending on the neutrino mass ordering. Thus, measurements of $v_{e}$ appearance have the sensitivity to determine the mass ordering, too. The $v_{e}$ appearance is observed with a $7.3 \sigma$ significance by the T2K experiment with data obtained with $6.6 \times 10^{20}$ POT in 2013[5]. This observation, 28 candidate events over $4.92 \pm 0.55$ background expectation, when combined with reactor measurements on $\sin \theta_{13}$ [1], enables us to put a constraint on possible $\delta_{\mathrm{CP}}$ value. Figure 2 shows the credible regions for $\sin ^{2} \theta_{13}$ and $\delta_{\mathrm{CP}}$ for $\mathrm{T} 2 \mathrm{~K}$-only and $\mathrm{T} 2 \mathrm{~K}+$ reactor combined analyses. The statistics is too low to make any conclusion, but the result looks interesting with the best-fit point at the maximum CP-violation point. More data are highly desired.

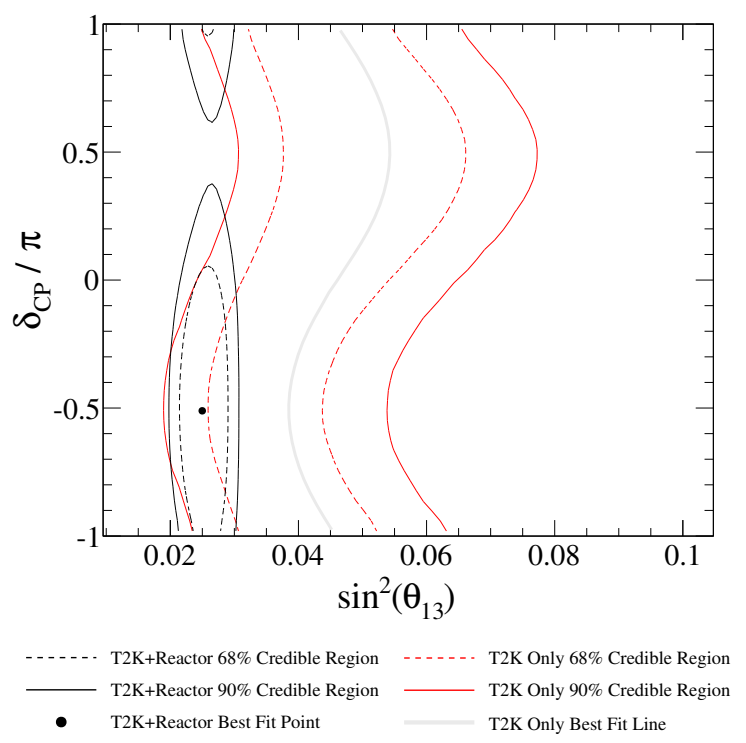

Figure 2: Credible regions for $\sin ^{2} \theta_{13}$ and $\delta_{\mathrm{CP}}$ for T2K-only and T2K+reactor combined analyses[2].

\section{Measurements with antineutrino}

Measurements with antineutrino are more difficult because the cross section is smaller by factor of 2 to 3 compared to that of neutrino. However, the measurement of appearance with antineutrino is important and indispensable to explore CP violation. The CPT theorem imposes that the disappearance rate should be same for neutrino and antineutrino. So the comparison of disappearance between neutrino and antineutrino would be a unique test of new physics. The unknown non-standard interaction with the Earth matter during the propagation may cause difference in the accelerator-based long-baseline experiments. Since 2014, T2K has been accumulating data with the muon antineutrino beam. The first result of antineutrino disappearance obtained with $2.3 \times 10^{20}$ POT is shown in Fig. 3 together with that obtained with the neutrino beam. The result is consistent with that observed with neutrinos. 


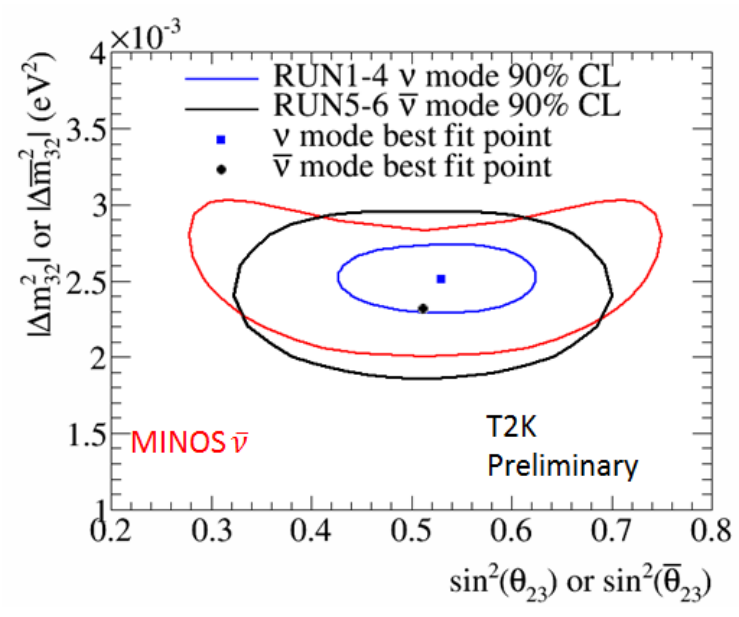

Figure 3: $90 \%$ C.L. for $\Delta m_{32}^{2}$ and $\sin ^{2} \theta_{23}$ obtained with T2K $6.6 \times 10^{20}$ POT neutrino-beam data and $2.3 \times 10^{20}$ POT antineutrino-beam data. MINOS antineutrino result[6] is overlaid.

\section{Near future prospects}

T2K has been accumulated $13 \%$ of the goal statistics so far. NOvA has just started and has $8 \%$ of the goal. What can be expected when these two experiments accumulated all the target data? The expected numbers of signal events at full statistics are $\sim 110 v_{e}$ and $\sim 20 \bar{v}_{e}$ for T2K and $\sim 70 v_{e}$ and $\sim 30 \bar{v}_{e}$ for NOvA. These numbers would be changed by $27 \%$ at most by the CP-violating term. The matter effect would change the numbers differently for T2K and NOvA because the baseline length and neutrino energy are different by about factor three for these two experiment. It is about $10 \%$ effect for T2K and $30 \%$ for NOvA. This difference, indeed, allows the sensitivities to $\mathrm{CP}$ violation and mass ordering be very much improved when results from the two experiment are combined. Figure 4 shows the sensitivities for $\mathrm{CP}$ violation and mass ordering both for individual experiments and combined case[7]. For favorable solutions, e.g., $\delta_{\mathrm{CP}}=-90^{\circ}$ and the normal mass ordering, either of the two experiments has a 90\% C.L. sensitivity for CP violation and NOvA has a good sensitivity to determine the mass ordering. When results are combined, sensitivities are enhanced and the mass ordering can be determined with $90 \%$ C.L. even for unfavorable parameter space. Therefore, at the end of these two experiments, we would expect some hints or even answer for $\mathrm{CP}$ violation and the mass ordering.

\section{Summary}

Since the discovery of neutrino oscillation and finite neutrino mass in 1998[8], our knowledge in this field has steadily evolved. We now know all the three mixing parameters and absolute values of the squared mass differences. What is remaining are the $\mathrm{CP}$ phase $\delta_{\mathrm{CP}}$, which is a key in the understanding of the matter-dominant universe, the value of $\theta_{23}$, which is found to be very close to the maximal mixing case and the order of the masses. The observation of $v_{e}$ appearance by T2K opened a way to access $\delta_{\mathrm{CP}}$. The precise measurement of $v_{\mu}$ disappearance showed that 

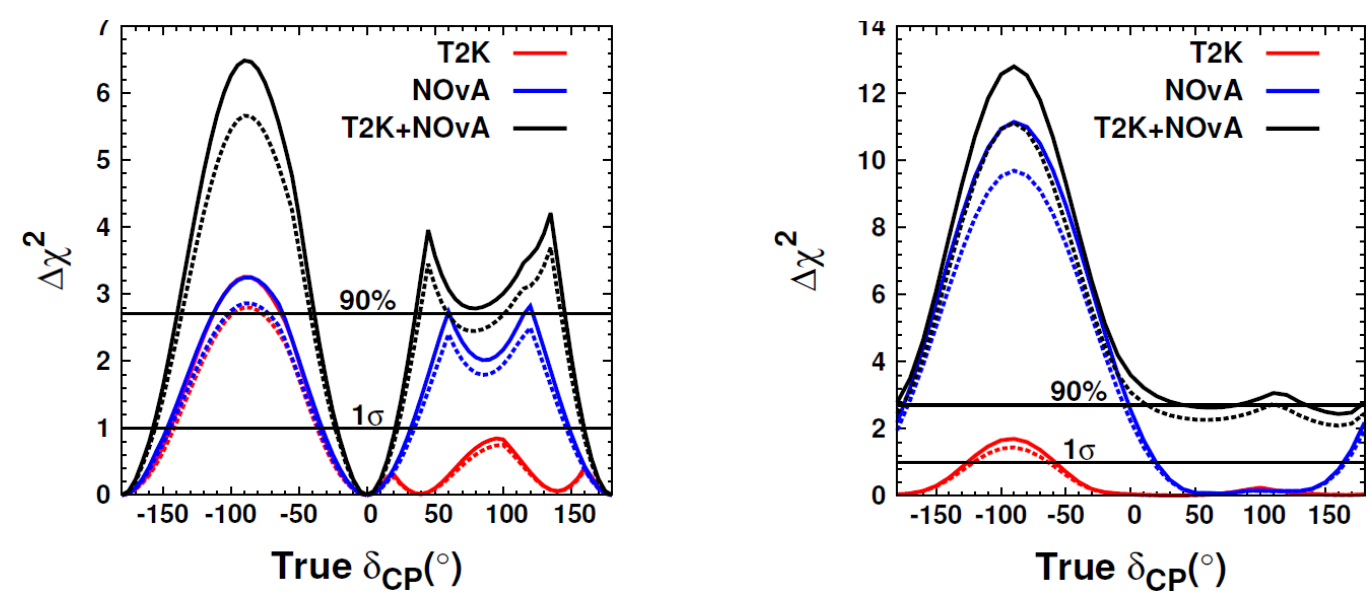

Figure 4: The sensitivity to the $\mathrm{CP}$-violation, i.e., non-zero $\sin \delta_{\mathrm{CP}}$, (left) and to the mass hierarchy discrimination (right) for various values of $\delta_{\mathrm{CP}}$ for T2K (red), NOvA (blue) and T2K and NOvA combined (black) [7]. Solid curves correspond to sensitivities with statistical errors only, while dashed ones additionally with projected systematic errors. These sensitivities are for the case of $\sin ^{2} \theta_{23}=0.5$ and the normal mass hierarchy.

$\theta_{23}=46^{\circ} \pm 3^{\circ}$. Accumulation of more data by $\mathrm{T} 2 \mathrm{~K}$ and NOvA may answer these open questions or further increase questions with unexpected observation in near future.

\section{References}

[1] K. A. Olive et al. Review of Particle Physics. Chin. Phys., C38:090001, 2014.

[2] K. Abe et al. Measurements of neutrino oscillation in appearance and disappearance channels by the T2K experiment with $6.6 \times 10^{20}$ protons on target. Phys. Rev., D91(7):072010, 2015.

[3] K. Abe et al. Evidence for the Appearance of Atmospheric Tau Neutrinos in Super-Kamiokande. Phys. Rev. Lett., 110(18):181802, 2013.

[4] N. Agafonova et al. Observation of tau neutrino appearance in the CNGS beam with the OPERA experiment. PTEP, 2014(10):101C01, 2014.

[5] K. Abe et al. Observation of Electron Neutrino Appearance in a Muon Neutrino Beam. Phys. Rev. Lett., 112:061802, 2014.

[6] P. Adamson et al. Measurement of Neutrino and Antineutrino Oscillations Using Beam and Atmospheric Data in MINOS. Phys. Rev. Lett., 110(25):251801, 2013.

[7] K. Abe et al. Neutrino Oscillation Physics Potential of the T2K Experiment. PTEP, 2015(4):043C01, 2014.

[8] Y. Fukuda et al. Evidence for Oscillation of Atmospheric Neutrinos. Phys. Rev. Lett., 81:1562-1567, 1998. 